\title{
Electrophoretic Mobility Shift Assay
}

National Cancer Institute

\section{Source}

National Cancer Institute. Electrophoretic Mobility Shift Assay. NCI Thesaurus. Code C19362.

A molecular biology technique used to detect the interaction of a DNA binding protein with its cognate binding sequence. Labeled DNAs were reacted with crude cell extracts and the complexes are run through a non-denaturing polyacrylamide gel. The migration of the labeled DNA through the gel will be slower by being bound. This shift in electrophoretic mobility indicates functional binding between the protein and the DNA. 